\title{
COMPORTAMENTO DO GIRASSOL EM RELAÇÃO A ACIDEZ DO SOLO (1)
}

\author{
MARIA REGINA GONCALVES UNGARO $\left({ }^{2,6}\right)$, JOSE ANTONIO OUAGGIO $\left({ }^{3,6}\right)$, \\ PAULO BOLLER GALLO ${ }^{4,6}$ ), SONIA CARMELA FALCI DECHEN $\left({ }^{5,6}\right)$, \\ FRANCISCO LOMBARDI NETO $\left({ }^{5}\right)$ e ORLANDO MELO DE CASTRO $\left({ }^{5,6}\right)$
}

\begin{abstract}
RESUMO
Em solo Podzólico Vermelho-Amarelo, foi instalado, no ano agrícola de 1981/82, um ensaio de rotação de culturas envolvendo milho, soja, mucuna-preta e girassol. Doze parcelas foram plantadas com girassol: algumas apresentaram germinação baixa, parte aérea pouco desenvolvida e com clorose generalizada nas folhas; sistema radicular com raiz pivotante encurvada e ramificaçoes secundárias pouco abundantes e grossas. As produçoes variaram conforme a intensidade desses sintomas. Com os resultados da análise de solo de cada parcela, obtiveram-se relaçoes lineares entre produção de grãos de girassol e pH $(\mathrm{r}=0,95)$ e saturação em bases do solo $(\mathrm{r}=0,92)$. Obtiveram-se ainda outras relaçoes, também lineares, entre altura de plantas, diâmetro dos capítulos e os índices de acidez do solo. Os resultados mostraram que o girassol também foi sensível à acidez do solo e que o critério do alumínio trocável, para a recomendaçđo de calagem, ño foi o mais adequado para esta planta.
\end{abstract}

Termos de indexação: girassol; Helianthus annuus; acidez; calagem; saturação em bases.

( $\left.{ }^{1}\right)$ Com auxílio parcial do projeto EMBRAPA/SAA. Apresentado no XIX Congresso Brasileiro de Ciencias do Solo, em Curitiba (PR), 19-24 de julho de 1983. Recebido para publicação em 19 de setembro de 1983.

$\left({ }^{2}\right)$ Seção de Oleaginosas, Instítuto Agronômico (IAC), Caixa Postal 28, 13100 - Campinas (SP).

( $\left.{ }^{3}\right)$ Seção de Fertilidade do Solo, IAC.

$\left({ }^{4}\right)$ Estaçao Experimental de Mococa, IAC.

(5) Seção de Conservaçżo do Solo, IAC.

$\left({ }^{6}\right)$ Com bolsa de suplementação do CNPq. 


\section{INTRODUÇÃO}

O girassol (Helianthus annuus L.) é uma oleaginosa produtora de excelente óleo comestível e com grande potencialidade de cultivo no território brasileiro, principalmente por ser pouco sensfvel ao fotoperiodismo e mais tolerante à seca e ao frio que culturas tradicionais, como milho e trigo. Por essas características, tem sido indicado sobretudo como cultura "da seca", de preferência em rotação com a de soja, do que resulta uma segunda produção de grãos, na mesma área, propiciando o uso da indústria de extração de óleos vegetais durante o período da entressafra da soja (UNGARO, 1978).

Por ser uma cultura ainda pouco difundida em nosso país, praticamente inexistem informaçðes a respeito das melhores condiçбes de solo para seu cultivo. Apesar de sua grande difusão em várias regires do mundo, muito pouco se conhece a respeito de suas exigências em $\mathrm{pH}$ e saturação em bases, visto que, na maior parte das vezes, ela é cultivada em solos com $\mathrm{pH}$ elevado ou ate mesmo em solos alcalinos. Os trabalhos de HORTENSTINE \& FISKELL (1961); BLAMEY (1975) e BLAMEY \& NATHANSON (1978) e a recente revisão de literatura de UNGARO (1983) têm demonstrado que o girassol é sensível à toxicidade provocada pelo excesso do alumínio no solo, apesar de ser conhecida a existência de diferenças de comportamento entre os cultivares em relação à tolerância a esse íon (FOY et alii, 1974).

O objetivo do presente trabalho foi identificar, mediante análise de solo, os fatores responsáveis pela baixa germinação, pequeno desenvolvimento da parte aérea e do sistema radicular, com clorose generalizada nas folhas e, ainda, a baixa produtividade de algumas parcelas de girassol em um ensaio de rotação de culturas envolvendo soja, milho, girassol e mucuna-preta. O sistema radicular, além de pouco desenvolvido, apresentava encurvamento da raiz principal, com poucas e grossas ramificaçס̄es secundárias.

\section{MATERIAL E MÉTODOS}

No ano agrícola de 1981/82, foi instalado um ensaio de rotação de culturas envolvendo girassol, milho, soja precoce e mucuna-preta, em solo classificado como Podzólico Vermelho-Amarelo, na Estação Experimental de Mococa, do Instituto Agronômico. Escolheu-se para a instalação do experimento, um local que estava em pousio havia anos. Retirou-se uma amostra composta de 15 subamostras, que, após análise, apresentou os seguintes resultados: $2,1 \%$ de matéria orgânica, $\mathrm{pH}$ em $\mathrm{H}_{2} \mathrm{O}=5,2 ; 0,3$ meq de $\mathrm{Al}^{3+} / 100 \mathrm{~cm}^{3} ; 1,5$ meq de $\mathrm{Ca}^{2+} / 100 \mathrm{~cm}^{3} ; 0,6$ meq de $\mathrm{Mg}^{2+} / 100 \mathrm{~cm}^{3} ; 104 \mu \mathrm{g} \mathrm{de}$ $\mathrm{K}^{+} /$mililitro; $4,1 \mu \mathrm{g}$ de $\mathrm{P} /$ mililitro. $\mathrm{O}$ ensaio foi instalado sem calagem, uma 
vez que, até o final de 1982, o critério do Instituto Agronômico não a recomendava para as condiçбes apresentadas por tal solo.

O ensaio era constituldo de 9 tratamentos e 4 repetiçסes, com um total de 36 parcelas de $8 \mathrm{~m} \times 12 \mathrm{~m}$, dispostas em delineamento estatístico de blocos ao acaso. No ano agrícola de $1981 / 82,12$ parcelas foram plantadas com girassol, cultivar IAC-Anhandy, no espaçamento de $1 \mathrm{~m}$ entre linhas e $0,2 \mathrm{~m}$ entre plantas. A adubação N-P-K no plantio foi na proporção de 20-60$-40 \mathrm{~kg} / \mathrm{ha}$ respectivamente de $\mathrm{N}, \mathrm{P}_{2} \mathrm{O}_{5}$ e $\mathrm{K}_{2} \mathrm{O}$, além de $40 \mathrm{~kg}$ de $\mathrm{N}$ por hectare em cobertura, na forma de sulfato de amônio, quarenta dias após o plantio.

Após a colheita das culturas "das águas", em março de 1982, foi feita uma amostragem de solo, em todas as parcelas do ensaio: retiraram-se 12 amostras simples para formar uma amostra composta por parcela, analisando-as seguindo os métodos propostos por RAIJ \& QUAGGIO (1983). As produçoes foram avaliadas em $30 \mathrm{~m}^{2}$ de área util das parcelas; antes da colheita, fez-se a medida da altura das plantas e do diâmetro dos capitulos.

\section{RESULTADOS E DISCUSSÃO}

Os resultados de análise de solo das parcelas onde se plantou girassol encontram-se no quadro 1 , juntamente com as produçסes correspondentes. Houve grande variação entre parcelas, principalmente em relação a alguns Indices de acidez do solo, como o pH, alumínio trocável, hidrogênio e saturação em bases. Isso demonstra que o terreno estava manchado e que, provavelmente, partes dele haviam recebido calagem anteriormente.

A influência do $\mathrm{pH}$ na produtividade do girassol pode ser vista na figura 1, que apresenta as relaçoes entre a produtividade em grãos e $\mathrm{opH}$ determinado em água (Figura $1 \mathrm{~A}$ ) e em solução de $\mathrm{CaCl}_{2}$ 0,01 $\mathrm{M}$ (Figura 1B). Observou-se melhor correlação entre produtividade e $\mathrm{pH}$ determinado em solução de $\mathrm{CaCl}_{2}$ 0,01 $\mathrm{M}$ do que em água, acontecendo o mesmo com a saturação em bases em relação à saturação em alumínio no solo. A figura 2 mostra que a saturação em bases (A) explicou melhor as variaçðes em produção do que a saturação em alumínio (B), pois houve aumentos de produtividade de até $32 \%$ apos a saturação em alumínio ser igual a zero, enquanto a saturação em bases mostrou-se linearmente correlacionada com a produção do girassol.

Esses resultados mostraram, ainda, que a quantidade de calcário calculada mediante o critério do alumínio trocável para a recomendação de calagem, como proposto por KAMPRATH (1970), ou mesmo como vinha sendo utilizado no Estado de São Paulo (QUAGGIO, 1983b), é insuficiente para 
QUADRO 1 - Caracteristicas químicas do solo das parcelas experimentais cultivadas com girassol e respectivas produçð̋es

\begin{tabular}{|c|c|c|c|c|c|c|c|c|c|c|c|}
\hline \multirow{2}{*}{ Parcela } & \multirow{2}{*}{$\begin{array}{l}\text { Maté- } \\
\text { ria or- } \\
\text { gânica }\end{array}$} & \multirow{2}{*}{$\mathbf{P}$} & \multicolumn{2}{|c|}{$\mathrm{pH}$} & \multirow{2}{*}{$\mathrm{H}^{+}$} & \multicolumn{4}{|c|}{ Cátions trocáveis } & \multirow{2}{*}{$\begin{array}{c}\text { Saturação } \\
\text { em } \\
\text { bases }\end{array}$} & \multirow{2}{*}{$\begin{array}{l}\text { Produ- } \\
\text { ção de } \\
\text { grãos }\end{array}$} \\
\hline & & & $\mathrm{H}_{2} \mathrm{O}$ & $\mathrm{CaCl}_{2}$ & & Al & $\mathrm{Ca}$ & $\mathbf{M g}$ & $\mathbf{K}$ & & \\
\hline no & $\%$ & $\mu \mathrm{g} / \mathrm{cm}$ & & & & & meq/ & & & $\%$ & $\mathrm{~kg} / \mathrm{ha}$ \\
\hline 01 & 2,4 & 5,5 & 4,7 & 4,1 & 5,1 & 0,7 & 0,8 & 0,4 & 0,15 & 21 & 1.153 \\
\hline 03 & 2,5 & 5,1 & 4,5 & 4,0 & 5,5 & 1,1 & 0,7 & 0,3 & 0,13 & 17 & 1.143 \\
\hline 06 & 2,4 & 6,5 & 4,7 & 4,1 & 5,5 & 0,8 & 0,8 & 0,4 & 0,15 & 20 & 1.127 \\
\hline 12 & 2,1 & 8,9 & 4,7 & 4,0 & 5,6 & 1,1 & 0,6 & 0,3 & 0,18 & 16 & 1.276 \\
\hline 15 & 2,1 & 6,9 & 5,9 & 5,4 & 2,2 & 0,0 & 2,5 & 1,4 & 0,16 & 65 & 2.497 \\
\hline 17 & 2,2 & 4,6 & 5,4 & 4,7 & 3,2 & 0,1 & 2,0 & 0,9 & 0,21 & 49 & 1.914 \\
\hline 21 & 2,5 & 4,1 & 5,0 & 4,6 & 3,5 & 0,1 & 1,3 & 0,9 & 0,13 & 40 & 2.152 \\
\hline 23 & 2,0 & 3,7 & 4,7 & 4,2 & 4,6 & 0,7 & 0,8 & 0,4 & 0,15 & 23 & 1.538 \\
\hline 27 & 2,0 & 9,4 & 5,6 & 5,1 & 2,2 & 0,0 & 1,8 & 1,2 & 0,14 & 59 & 2.272 \\
\hline 33 & 2,1 & 12,6 & 5,8 & 5,1 & 2,4 & 0,0 & 2,1 & 1,2 & 0,14 & 60 & 2.051 \\
\hline 35 & 2,7 & 17,2 & 5,6 & 5,0 & 2,9 & 0,0 & 2,5 & 1,1 & 0,21 & 58 & 1.920 \\
\hline 36 & 1,8 & 5,9 & 6,0 & 5,5 & 2,0 & 0,0 & 2,6 & 1,3 & 0,13 & 67 & 2.854 \\
\hline
\end{tabular}

suprir as exigências do girassol, e sugerem que o método baseado na correlação entre o $\mathrm{pH}$ e a saturação em bases, como proposto por QUAGGIO (1983a), talvez seja mais adequado. Isso discorda da opinião de BLAMEY \& NATHANSON (1978) que concluíram que a dose de calcário recomendada para essa cultura não deveria exceder a quantidade necessária para eliminar o alumínio trocável do solo.

As figuras 3 e 4 mostram que a altura de plantas e o diâmetro de capítulos também são influenciados pela acidez do solo, e que as variaçoes observadas no experimento estiveram relacionadas com o $\mathrm{pH}$ e com a saturação em bases do solo, do mesmo modo que a produtividade.

Esses resultados revelaram que o girassol é planta muito sensível à acidez do solo, o que está de acordo com os resultados de HORTENSTINE \& FISKELL (1961), BLAMEY (1975) e BLAMEY \& NATHANSON (1978). Além disso, o cultivar IAC-Anhandy, utilizado no ensaio, é originário de seleção massal no 'Peredovick', sendo este, segundo FOY et alii (1974), pouco tolerante à toxicidade provocada pelo excesso de alumínio trocável no solo. 

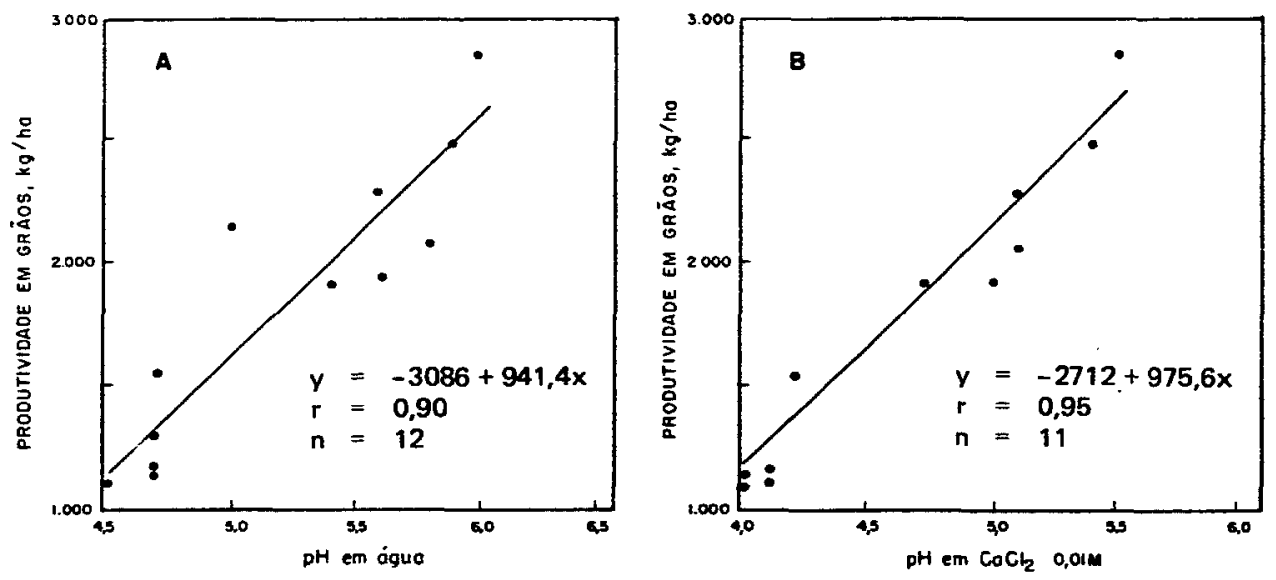

FIGURA 1 - Relação entre pH em $\mathrm{H}_{2} \mathrm{O}$ (A) e pH em solução 0,01 $\mathrm{M}$ de $\mathrm{CaCl}_{2}$ (B) e produtividade em grãos de girassol.
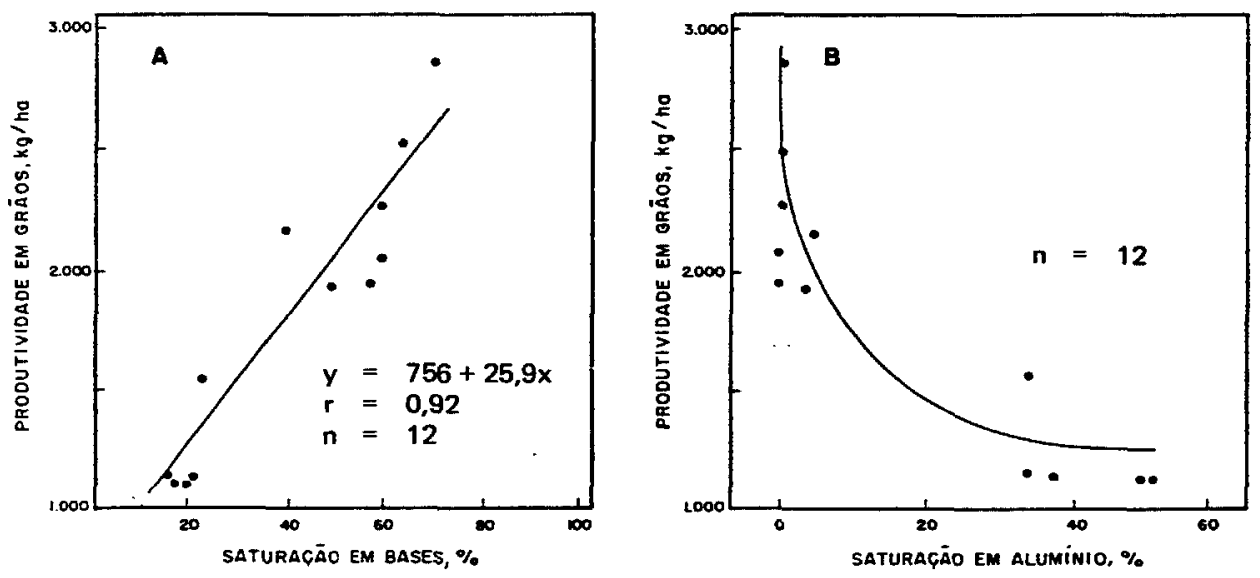

FIGURA 2 - Relação entre saturaçđo em bases (V\%) (A) e saturação em alumínio (Al\%) (B) com produtividade de grãos de girassol. 

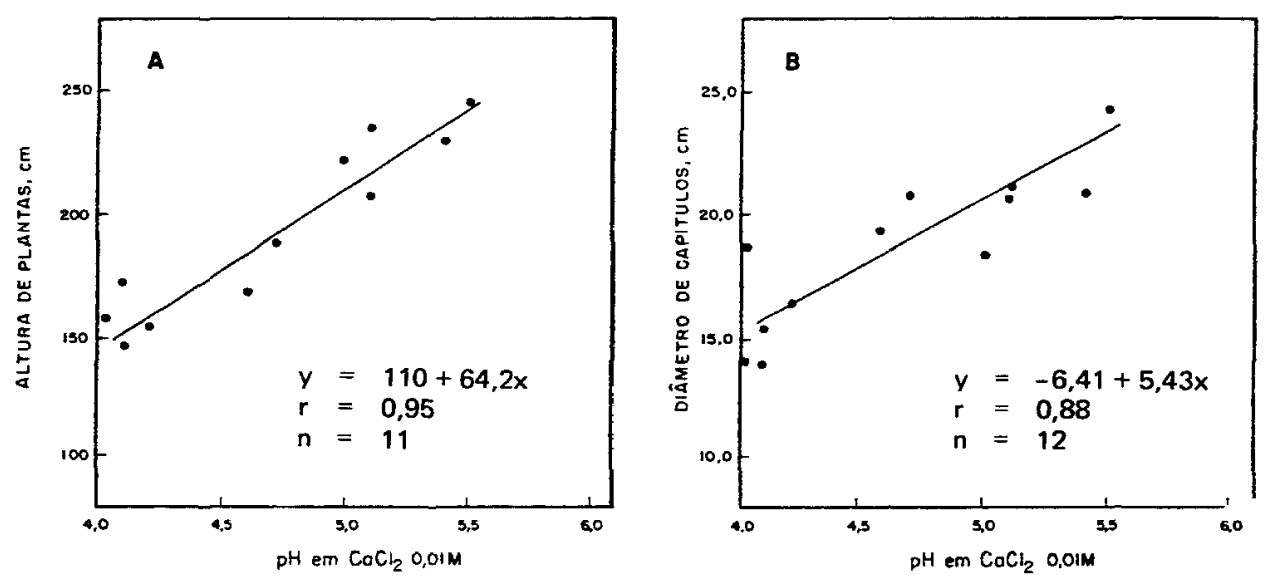

FIGURA 3 - Relação entre pH em solução $0,01 \mathrm{M}$ de $\mathrm{CaCl}_{2}$ com altura de plantas (A) e diâmetro de capítulos de girassol (B).
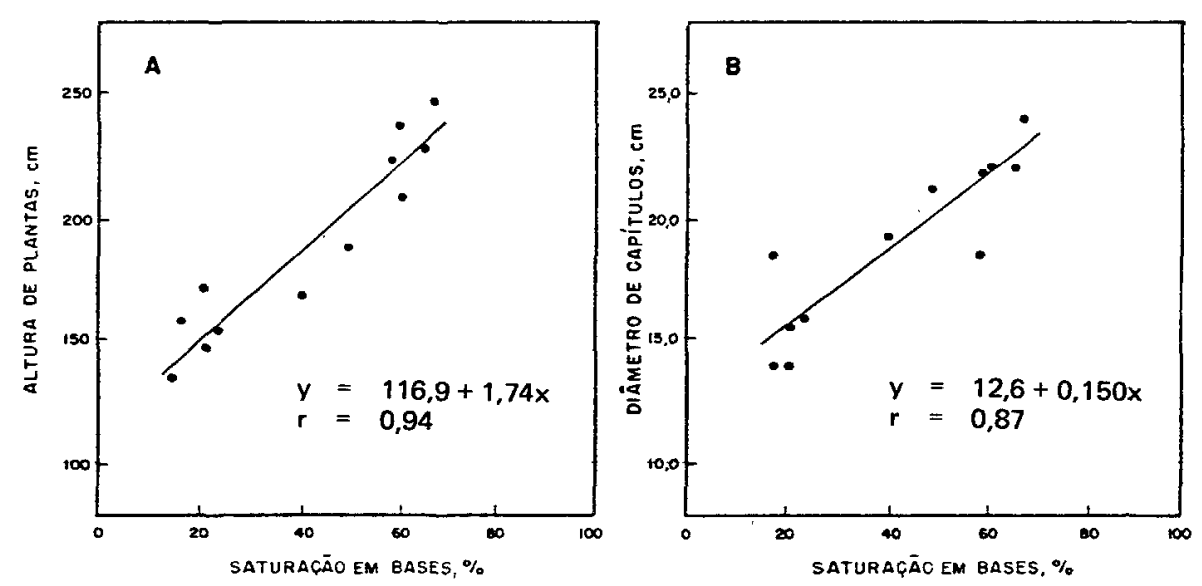

FIGURA 4 - Relação entre saturação em bases (V\%) e altura de plantas (A) e diâme tro de capitulos de girassol (B). 


\section{CONCLUSÕES}

Com os resultados obtidos nesse experimento, pode-se concluir que, para o girassol IAC-Anhandy:

a) a fraca germinação, pequeno desenvolvimento da parte aérea e clorose generalizada nas folhas, sistema radicular pouco desenvolvido, com encurvamento da raiz principal, com poucas e grossas ramificaçðes, foram devidos à acidez do solo e nem todos foram explicados pela toxicidade provocada pelo alumínio;

b) apesar de o girassol ter-se mostrado sensível ao excesso de alumínio trocável no solo, este parece não ser o critério mais adequado para a tecomendação de calagem para essa cultura, mas, sim, o baseado na correlação entre o pH e a saturação em bases.

\section{SUMMARY}

\section{SUNFLOWER RESPONSE TO SOIL ACIDITY CONDITIONS}

A crop rotation experiment involving corn, soybean, sunflower, and green manure was carried out in a Red-Yellow Podzolic soil. In some of the 12 plots with sunflower the seed germination was poor and the plants showed reduced growth, with leaf chlorosis, curved main roots, and fewer and thicker secondary roots. Yield was negatively correiated with the intensity of these symptoms. The grain yield was highly correlated with soil $\mathrm{pH}(\mathrm{r}=0.95)$ and soil base saturation $(\mathrm{r}=0.92)$. Plant height and head diameter also showed linear relationship with those soil indices. The results obtained show that sunflower is very sensitive to soil acidity, and that the exchangeable aluminum criterion is inadequate for determining lime requirement for sunflower.

Index terms: sunflower; Helianthus annuus; soil acidity; liming; base saturation.

\section{REFERÊNCIAS BIBLIOGRÁFICAS}

BLAMEY, F, P. C. Amelioration of an acid Avalon medium sandy loam and effects on the growth of sunflowers (Helianthus annuus L.). Crop Production, Budapeste, 4:75-79, 1975.

\& NATHANSON, K. Relatioships between aluminum toxicity and sunflower yields on an Avalon medium loam. Sunflower News, Zevenaar, 2:6-12, 1978.

FOY, C. D.; ORELLANA, R. G.; SCHWARTZ, J. W. \& FLEMING, A. L. Response of sunflower genotypes to aluminum in acid soil and nutrient solution. Agronomy Journal, Madison, 66:293-296, 1974. 
HORTENSTINE, C. C. \& FISKELL, J. G. A. Effect of aluminum on sunflower growth and uptake of boron and calcium from nutrient solution. Soil Science Society of America Proceedings, Madison, 25:304307, 1961.

KAMPRATH, E. J. Exchange aluminum as a criterion for liming leached mineral soils. Soil Science Society of America Proceedings, Madison, 34: 252-254, 1970.

QUAGGIO, J. A. Critérios para calagem em solos do Estado de São Paulo. Piracicaba, Escola Superior de Agricultura "Luiz de Queiroz", 1983a. 76f. Tese. (Mestrado)

Métodos de laboratório para a determinação da necessidade de calagem em solos. In: REUNIÃO BRASILEIRA DE FERTILIDADE DO SOLO, 15., Campinas, 1982, acidez e calagem no Brasil: simpósio. Campinas, Sociedade Brasileira de Ciência do Solo, 1983b. p. 33-48.

RAIJ, B. van \& QUAGGIO, J. A. Métodos de análise de solo para fins-de fertilidade. Campinas, Instituto Agronômico, 1983. 31 p. (Boletim técnico, 81)

UNGARO, M.R.G. Calagem em girassol. In:REUNIÃO BRASILEIRA DE FERTILIDADE DO SOLO, 15., Campinas, 1982. Simpósio sobre acidez e calagem no Brasil. Campinas, Sociedade Brasileira de Ciência do Solo, 1983. p. $277-281$.

Instruçðes para a cultura do girassol. Campinas, Instituto Agronômico, 1978. 15p. (Boletim técnico, 212) 